\title{
Scrotal recurrence of germ cell tumour in a non-violated scrotum
}

\author{
Jenny Li;i Nicholas Power, MD² \\ ISchulich School of Medicine \& Dentistry, Western University, London, ON, Canada; 2Division of Urology, London Health Sciences Centre, Western University, London, ON, Canada
}

Cite as: Can Urol Assoc J 2016;10(11-12):E388-91. http://dx.doi.org/10.5489/cuai.3505

Published online November 10, 2016.

\section{Abstract}

Testicular cancer is the most common cancer diagnosis in males aged 15-30 years. For over a century, radical inguinal orchiectomy has been the standard of care for initial treatment of testicular cancer. This approach is preferred over trans-scrotal interventions, in an effort to avoid tumour seeding, spermatic cord invasion, and disturbance to lymphatic drainage. Scrotal violation is defined as any trans-scrotal intervention that may impact spread of disease in testicular cancer, including scrotal orchiectomy, fine-needle aspiration, and testicular biopsy. Studies have shown statistically significant differences in local recurrence rates between patients who undergo the standard inguinal surgical approach and cases with scrotal violation.

Over $95 \%$ of testicular cancers are curative, often with surgery alone. Recurrence of disease is divided into two categories: local and distant sites. Local recurrence of testicular cancer involves the scrotal and inguinal regions, including superficial inguinal lymph nodes. More commonly, local recurrence is seen in cases of testicular cancer with scrotal violation. We describe a case of local recurrence of testicular cancer in a non-violated scrotum, a finding that has not been previously reported in the literature.

\section{Introduction}

Testicular cancer is the most common cancer diagnosis among males between the ages of 15 and 30. In 2015, an estimated 1050 individuals were diagnosed with testicular cancer in Canada. ${ }^{1}$ With current treatment modalities, testicular cancers have an excellent prognosis; the five-year survival rates is well over $95 \% .^{2}$ Surgery is not only therapeutic and diagnostic, but often curative. ${ }^{2,3}$

Radical inguinal orchiectomy with high ligation of the spermatic cord is the standard of care in initial treatment of testicular cancer. ${ }^{4}$ The inguinal approach is preferred over scrotal manipulations to prevent disturbance to lymphatic drainage, and thus avoiding potential spread of disease. ${ }^{5}$ Violation of the scrotum is defined as any trans-scrotal intervention that can impact tumour seeding. ${ }^{5}$ Scrotal violation in relation to testicular cancer can include open testicular biopsy, fine-needle aspiration, trans-scrotal orchiectomy or previous repair of inguinal hernias, and hydrocolectomies. ${ }^{4,5}$ In such cases, a variety of adjuvant treatments, including hemiscrotectomy, inguinal lymph node dissection, local radiation, and chemotherapy, have been suggested. ${ }^{4}$ These subsequent treatments are aimed at preventing recurrence of disease and can often adversely affect patient morbidity.

Testicular cancer can recur both locally and at distant sites. Local recurrence involves disease in the scrotal or inguinal regions, including superficial lymph nodes. ${ }^{4}$ Distant recurrence includes all other sites, including biochemical recurrence seen by a rise in serum tumour markers. ${ }^{4,5}$ In a review by Capelouto et al, local recurrence rates for testicular cancer were significantly different between patients who underwent inguinal orchiectomies $(0.4 \%)$ and scrotal violation patients $(2.9 \%) .{ }^{4}$ Meta-analysis of 1182 cases showed an increased rate of local recurrence in scrotal violation patients, especially for individuals presenting with stage I disease. The majority of scrotal violation patients underwent adjuvant therapy to prevent further recurrence. ${ }^{4}$

We present a case of testicular cancer with local recurrence of testicular malignancy in a non-violated scrotum, a finding that has not been previously reported in the literature.

\section{Case report}

A 23-year-old male underwent a right orchiectomy for a mixed non-seminomatous germ cell tumour (NSGCT) $(40 \%$ seminoma, $30 \%$ embryonal carcinoma, $20 \%$ yolk sac tumour, $10 \%$ teratoma). Pathology showed a pT3 mass with negative surgical margins. Serum tumour markers remained elevated postoperatively. A subsequent computerized tomography (CT) scan of the abdomen and pelvis showed a large confluence of nodes in the inter-aortocaval region (Fig.1). The initial clinical stage and classification of disease was IIC (pT3N3M0S2) and IGCCCG intermediate prognostic group. The patient had planned primary therapy with four cycles of bleomycin/etoposide/cisplatin (BEP) chemotherapy. Tumour markers fell appropriately, but the patient's abdominal mass on examination was larger. After his third cycle of chemotherapy, repeat CT images revealed significant interval progression of disease (Fig. 2). After recognizing 


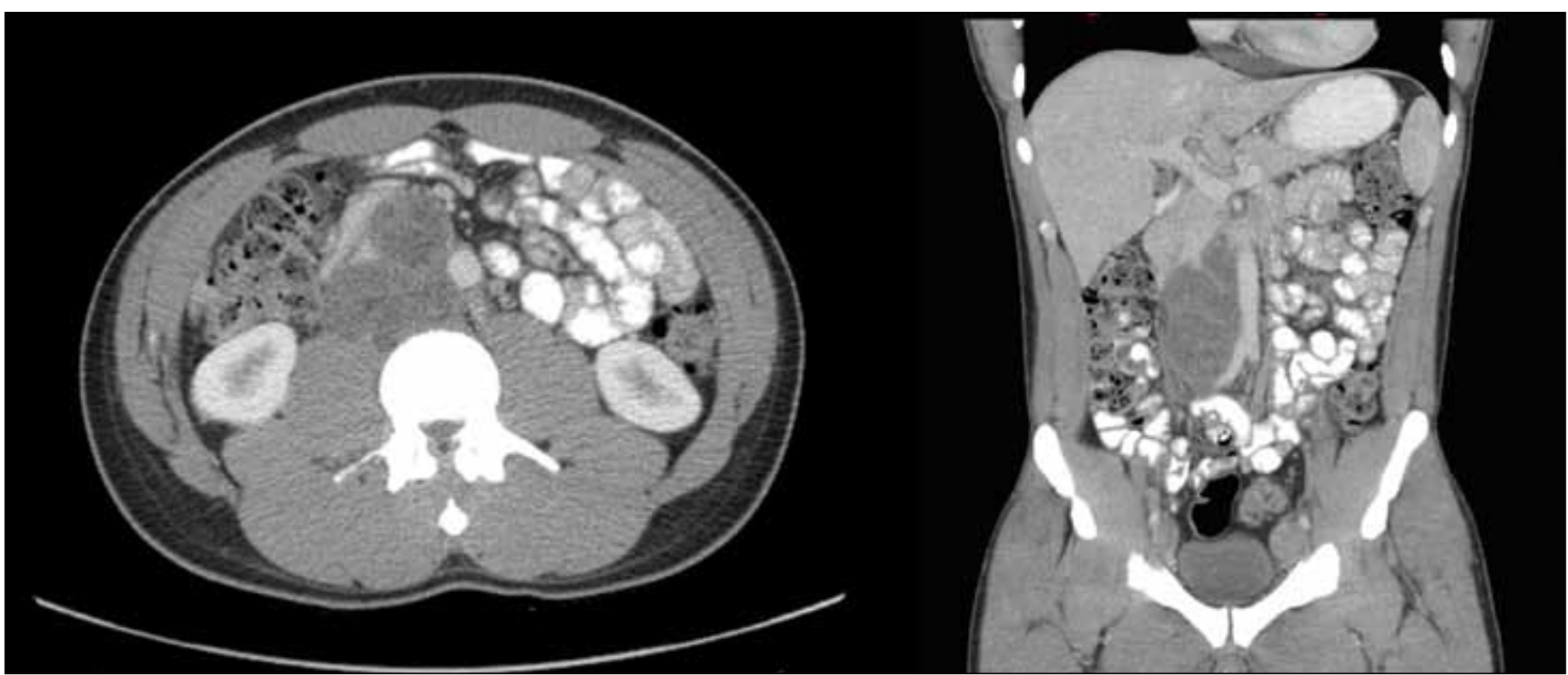

Fig.1. Axial and coronal contrast-enhanced computed tomography images a demonstrating retroperitoneal mass with a large confluence of nodes in the aorta-caval region, measuring approximately $6.0 \times 12.0 \times 6.0 \mathrm{~cm}$.

growing teratoma syndrome (GTS) in this patient, he was counselled to undergo urgent full bilateral retroperitoneal lymph node dissection (RPLND). The retroperitoneal tumour and surrounding lymph nodes were successfully resected. Pathology of the retroperitoneal mass showed all elements to be consistent with mature teratoma. Post-excision of the mass, tumour markers were within normal limits.

Seven months after his RPLND, the patient presented back to hospital with a $1 \mathrm{~cm}$ left-sided scrotal mass. The patient had noted that it had slightly increased in size over the course of three months. Scrotal ultrasound revealed a solid vascularized lesion with cystic components within the soft tissues of the superior left hemiscrotum, corresponding to the palpable abnormality (Fig. 3). The mass was resected without complication. Surprisingly, pathology revealed the specimen to be composed entirely of mature teratoma. Margins were negative. The patient is currently on surveillance and has remained disease-free for six months.

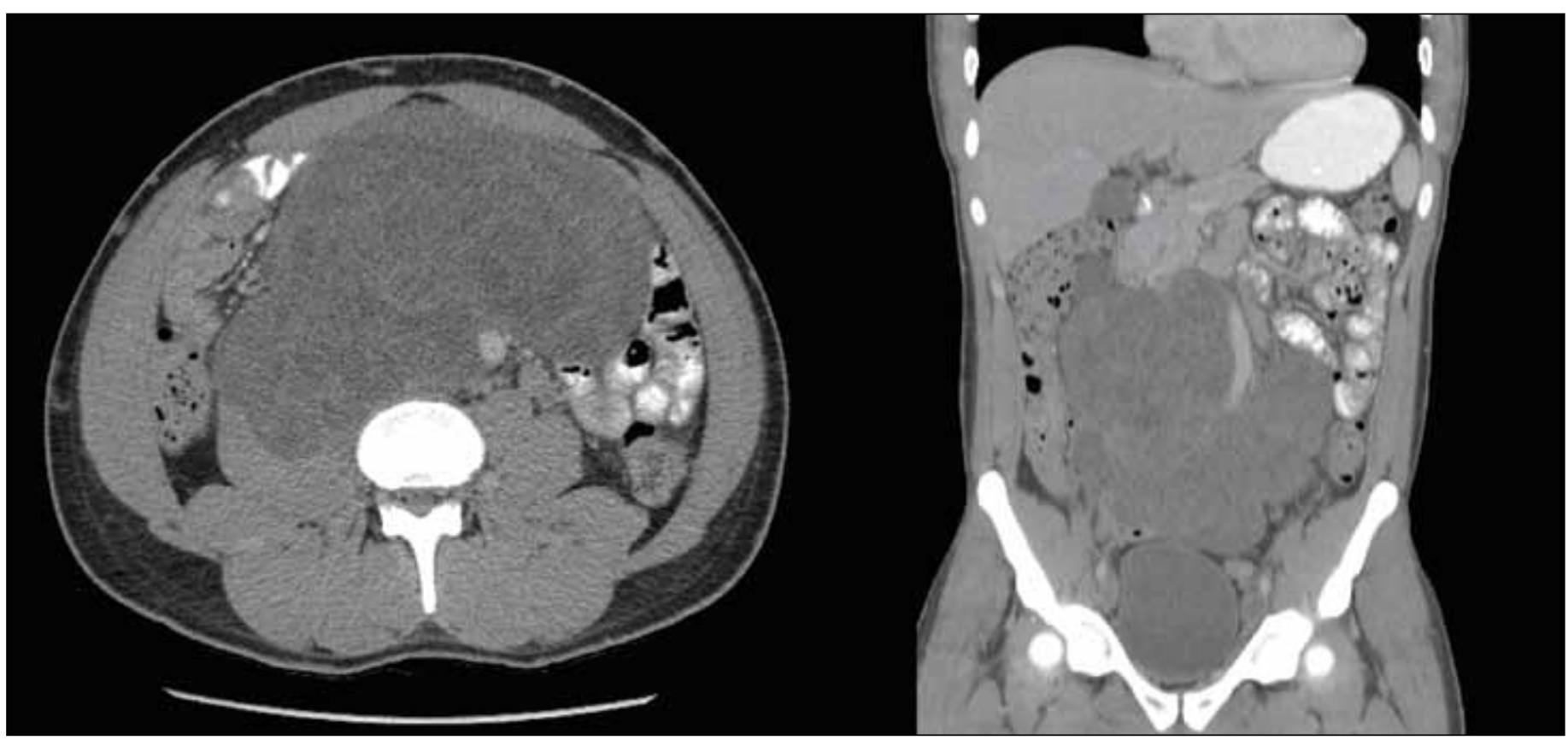

Fig.2. Rapid interval progression of retroperitoneal mass on axial and coronal contrast-enhanced computed tomography images over the course of three months. 


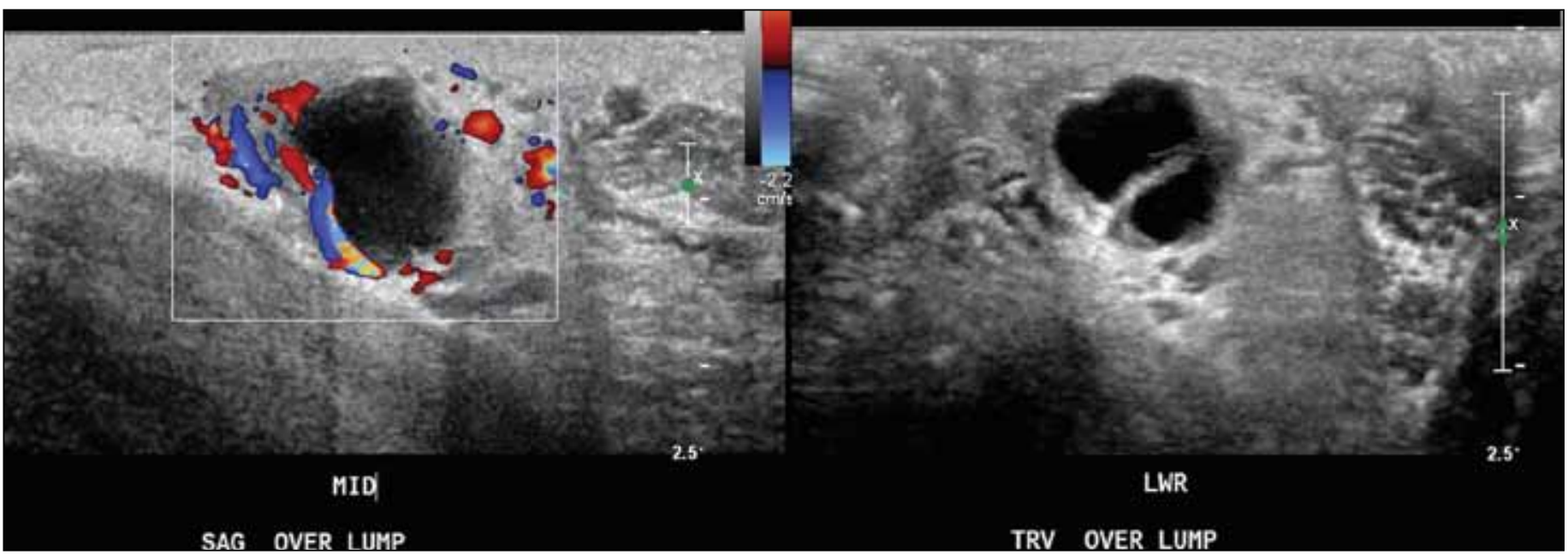

Fig. 3. An irregular, ovoid nodule in the left hemiscrotum with both solid and cystic components, measuring $1.8 \times 1.7 \times 1.1 \mathrm{~cm}$ on scrotal ultrasound. Significant flow was demonstrated in the solid portions using Doppler imaging.

\section{Discussion}

GTS is a rare clinical diagnosis found in $1.9-7.6 \%$ of patients with testicular NSGCT. ${ }^{6}$ These patients usually present with growing metastatic masses during or after chemotherapy. GTS is defined by three criteria: (1) new or enlarging masses despite ongoing chemotherapy treatment; (2) normal serum tumour markers alpha-fetoprotein (AFP) and beta human chorionic gonatropin (beta-hCG); and (3) histology of resected specimens exclusively showing mature teratoma. Complete surgical resection is the current gold standard treatment for GTS. Indeed, with surgery, the disease is usually curative. ${ }^{6,7}$ Although uncommon, GTS has been previously reported in the literature. ${ }^{8,9}$

Testicular cancer tends to spread through the lymphatic system, with the first site of metastasis usually to the para-aortic lymph nodes. ${ }^{2,3}$ Common sites of distant invasion include the liver, lung, and brain. ${ }^{2}$ Our patient had a local recurrence of teratoma in the scrotum less than a year after radical inguinal orchiectomy for a NSGCT in a non-violated scrotum.

In our patient, the finding of local recurrence could be attributed to the completeness of surgical resection. Although there were negative surgical margins on the pathology report of the orchiectomy specimen, it is possible that microscopic residual tumour cells were left in the scrotum at the original surgery.

It is also possible that the local recurrence of teratoma is related to the patient's concurrent diagnosis of GTS. In a study by Nativ et al, flow cytometric nuclear DNA analysis of tissue blocks from patients with findings of post-chemotherapy residual mature teratoma showed aneuploidy, demonstrating that mature teratoma cells are still prone to malignant behaviour. ${ }^{10}$ Although mature teratoma is benign in phenotype, its ability to metastasize has been well-established in the literature. ${ }^{11}$ Pure primary teratoma of the testis has been shown to metastasize after initial orchiectomy. ${ }^{11}$
The mechanism behind this spread of disease is likely related to pluripotent stem cells metastasizing and giving rise to well-differentiated teratoma cells through somatic differentiation rather than mature well-differentiated teratoma cells spreading to local and distant sites. ${ }^{12}$

Research on mechanisms of local recurrence of testicular cancer in a non-violated scrotum is limited. However, clinically important information for testicular cancer patients can be drawn from this case report. We would advise close surveillance of post-treatment patients, with serial imaging to monitor for local/distant recurrence. In addition, for patients with $\geq$ pT3 NSGCT primary tumours subsequently diagnosed with GTS, individuals should be counselled on self-examination of the scrotum and remaining testicle. Any changing abnormality should be aggressively resected.

Competing interests: Ms. Li reports no competing personal or financial interest. Dr. Power has participated in clinical trials with Argos Therapeutics.

This paper has been peer-reviewed.

\section{References}

1. Canadian Cancer Society's Advisory Committee on Cancer Statistics. Canadian Cancer Statistics 2015. Toronto, ON: Canadian Cancer Society; 2015. [May 2015, adapted June 2015]. Available at http:// www.cancer.ca/en/cancer-information/cancer-101/canadian-cancer-statistics-publication/? region=0n. Accessed October 6, 2016

2. Dearnaley D, Huddart R, Horwich A. Regular review: Managing testicular cancer. BMJ 2001;322:1583-8. http://dx.doi.org/10.1136/bmi.322.7302.1583

3. Guidelines on the management of adult testicular cancer. Clin Oncol (R Coll Radiol) 2000;12:S173-210.

4. Capelouto CC, Clark PE, Ransil BJ, et al. A review of scrotal violation in testicular cancer: Is adjuvant local therapy necessary? J Urol 1995; 153:981-5. http://dx.doi.org/10.1016/S0022-5347(01)67617-1

5. Aki FT, Bilen CY, Tekin MI, et al. Is scrotal violation per se a risk factor for local relapse and metastases in stage I non-seminomatous testicular cancer? Urology 2000;56:459-62. http://dx.doi.org/10.1016/ S0090-4295(00)00692-0 
6. Gorbatiy V, Spiess PE, Pisters LL. The growing teratoma syndrome: Current review of the literature. Indian J Urol 2009;25:186-9. http://dx.doi.org/10.4103/0970-1591.52910

7. Logothetis $\mathrm{CJ}$, Samuels ML, Trindade A, et al. The growing teratoma syndrome. Cancer 1982;50:1629-35. http://dx.doi.org/10.1002/1097-0142(19821015)50:8<1629::AIDCNCR2820500828>3.0.C0;2-1

8. Spiess PE, Kassouf W, Brown GA, et al. Surgical management of growing teratoma syndrome: The M. D. Anderson Cancer Centre experience. J Urol 2007;177:1330-4. http://dx.doi.org/10.1016/i. juro.2006.11.086

9. Inaoka $\mathrm{T}$, Takahashi $\mathrm{K}$, Yamada $\mathrm{T}$, et al. The growing teratoma syndrome secondary to immature teratoma of the ovary. Eur Radiol 2003;13:2115-8. http://dx.doi.org/10.1007/s00330-002-1733-3
10. Nativ 0 , Shairawi I, Leibovitch I, et al. The malignant potential of post-chemotherapy residual mature teratoma for disseminated non-seminomatous testicular tumours. Eur Urol 1993;26:216-18.

11. Simmonds PD, Lee AH, Theaker JM, et al. Primary pure teratoma of the testis. J Urol 1996;155:939-42. http://dx.doi.org/10.1016/S0022-5347(01)66352-3

12. Herr HW, LaQuaglia MP. Management of teratoma. Urol Clin North Am 1993;26:145-52.

Correspondence: Ms. Jenny Li, LHSC Victoria Hospital, London ON Canada; jennyli1500@gmail.com 